\title{
Suitability of BPM Simulation for Silicon Photonics
}

\begin{abstract}
Chenglin $\mathrm{Xu}^{1}$, Member IEEE, Evan Heller ${ }^{1}$, Mayank Bahl ${ }^{1}$, Rob Scarmozzino ${ }^{1}$, Kai-Ning $\mathrm{Ku}^{2}$, Ying Zhou ${ }^{1}$ and Tungyu Su${ }^{3}$, Po-Chih Chang ${ }^{2}$, Chen-Yu Lin ${ }^{2}$, Shang-Chun Chen², Chih-Lin Wang ${ }^{2}$, Chien-Chung Lin ${ }^{2}$, Senior Member IEEE
\end{abstract}

\begin{abstract}
Validated both numerically and experimentally, Beam Propagation Method (BPM) has been proven to be a very efficient and reasonably accurate simulation approach for certain silicon photonics (SiP) devices. This paper clears up some misunderstanding in SiP community that BPM is not suitable for SiP devices.
\end{abstract}

Index Terms - BPM, FDTD, EME, Silicon photonics, MMI, AWG, SSC, directional coupler.

\section{INTRODUCTION}

$\mathrm{V}$ ector beam propagation method (BPM) has been widely used to simulate fiber and waveguide devices efficiently since its invention 30 years ago [1,2]. The method makes certain approximations in the governing equation, which leads to fast simulation times and small memory requirements, while still working well under certain conditions, such as slowlyvarying structures with negligible backward reflection. For the high index contrast devices of silicon photonics ( $\mathrm{SiP})$, vector BPM, especially full-vector BPM [3] (FV-BPM), is not unconditionally stable, and may experience problems due to accumulated and amplified numerical noise if some modal parameters are not properly chosen. This created a wrong impression that BPM is not suitable for SiP. To correct this misunderstanding, we will first explore the suitable scope of BPM applications from the theoretical point of view and study the characteristics of $\mathrm{SiP}$ waveguides. Then we will apply BPM to several common silicon photonics devices and validate it against more rigorous numerical methods, such as the FiniteDifference Time-Domain Method (FDTD) and Eigen Mode Expansion (EME). We will also compare the simulation results with available experimental results.

\section{THEORETICAL BACKGROUND}

The equation that FV-BPM solves is the slowly-varying unidirectional wave equation [3],

$$
j 2 n_{0} k \frac{\partial}{\partial z}\left(\begin{array}{l}
F_{x} \\
F_{y}
\end{array}\right)=\boldsymbol{P} \overrightarrow{\boldsymbol{F}}=\left(\begin{array}{ll}
P_{x x} & P_{x y} \\
P_{y x} & P_{y y}
\end{array}\right)\left(\begin{array}{l}
F_{x} \\
F_{y}
\end{array}\right)
$$

Manuscript received on September 17, 2021. Kai-Ning Ku, Po-Chih Chang, Chen-Yu Lin, Shang-Chun Chen, Chih-Lin Wang, and Chien-Chung Lin would like to thank the financial support by the MOEA of Taiwan under contract No. 106-EC-17-D-11-1475.

${ }^{1}$ Chenglin $\mathrm{Xu}$, Evan Heller, Mayank Bahl, Rob Scarmozzino, and Ying Zhou are with Synopsys Optical Solution Group, Ossining, NY 10562 USA.
Here $F_{x}(x, y)$ and $F_{y}(x, y)$ are the envelopes of the transverse electric or magnetic field excluding the fast oscillating phase $e^{-j k n_{0} z}$. The expression of the operators $P_{x x}, P_{y y}, P_{x y}$, and $P_{y x}$, are defined in [3].

The finite difference solution to the above BPM equation is

$$
\vec{F}(z+\Delta z)=\frac{1-j 2 n_{0} k \Delta z(1-\alpha) P}{1+j 2 n_{0} k \Delta z \alpha P} \vec{F}(z)
$$

where $0 \leq \alpha \leq 1 \alpha$ is a scheme parameter, $n_{0}$ is the reference refractive index, and $k$ is the wavenumber in free space.

The above equation is a full vector equation because any of the other four field components can be represented by any pair of components, either $E_{x} \& E_{y}$, or $H_{x} \& H_{y}$, or $E_{z} \& H_{z}$. Therefore, "BPM equation is not full vector" is another misunderstanding to be corrected.

Surely, the BPM equation is an approximation and it comes with the following assumptions:

(1) The unidirectional wave equation determines that BPM can only trace forward waves and any backward reflection is ignored. Hence it is not applicable to structures with strong backward reflection, though bidirectional BPM can handle some limited cases [4].

(2) The paraxial approximation limits BPM to modeling beams with a relatively small divergence angle and a field containing modes with effective indices close to the reference index $n_{0}$. The wide-angle BPM $[5,6]$ can relax this limitation with extra computational effort.

(3) The envelope approximation excludes the fastoscillating phase, so it should be applied only to slowly varying structures. A dramatically changing structure causes scattering with a fast-oscillating envelope and some of it going backward.

Because of these approximations, FV-BPM sometimes suffers instability problems due to numerical noise generated by the field discontinuity at interfaces and especially singularities at corners. It has been proven mathematically that the numerical noise excites pairs of complex conjugates modes, even for lossless waveguides [7]. The pairs then propagate in opposite

${ }^{2}$ Kai-Ning Ku, Po-Chih Chang, Chen-Yu Lin, Shang-Chun Chen, Chih-Lin Wang, and Chien-Chung Lin are with ITRI, Hsinchu, Taiwan.

${ }^{3}$ Tungyu Su is with Synopsys Photonic Solution, Hsinchu, Taiwan.

Corresponding e-mail: chenglin@ synopsys.com. 
directions, the one propagating backward grows exponentially as the other propagates forward.

To make FV-BPM stable, a scheme parameter $\alpha>0.5$ is usually introduced to suppress the noise. As a drawback, larger scheme parameters introduce an artificial loss which can cause the total power not conserved.

In the case of weak polarization coupling, the full-vector equation (1) can be separated into two decoupled semi-vector equations by assuming $P_{x y}=P_{y x}=0$.

$$
j 2 n_{0} k \frac{\partial}{\partial z} F_{x}=P_{x x} F_{x}
$$

for TE wave, and

$$
j 2 n_{0} k \frac{\partial}{\partial z} F_{y}=P_{y y} F_{y}
$$

for TM wave.

Because only discontinuities are involved, this semi-vector BPM (SV-BPM) is much more stable than FV-BPM, and $\alpha=0.5$ can be used in most cases to maintain power conservation.

In the weak polarization case, the two semi-vector equations can be further simplified as a single scalar equation by assuming $P_{x x}=P_{y y}=P$.

$$
j 2 n_{0} k \frac{\partial}{\partial z} \psi=P \psi
$$

The scalar BPM (S-BPM) is unconditionally stable and power is conserved with $\alpha=0.5$, in the Crank-Nicolson scheme.

Naturally, the question is when should full-vector, semivector, or scalar BPM be used, especially for SiP with high index contrast?

\section{MODAL CHARACTERISTICS OF SIP WAVEGUIDES}

To answer the above commonly asked question, let's first investigate the modal characteristics, which is the foundation of any kind of waveguide analysis.

In general, two types of $\mathrm{SiP}$ waveguides, large core rib/ridge waveguide and small core silicon wire, are widely used. Their modes can be calculated by the mode solver built-in to BeamPROP BPM [8], which is based on imaginary-distance BPM [9] and correlation method [10].

\section{A. Large core rib/ridge waveguide}

Regardless of its large core, the rib/ridge waveguide is a weakly guided waveguide due to its weak confinement in the lateral direction.

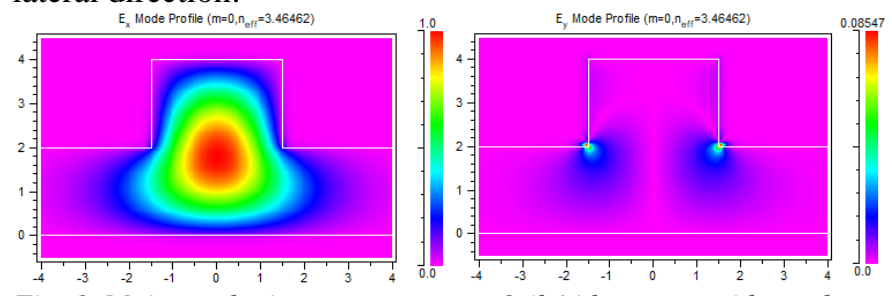

Fig. 1. Major and minor components of rib/ridge waveguide mode

Shown in Fig. 1 is the full-vector fundamental mode of a $\mathrm{rib} /$ ridge waveguide with $3 \mu \mathrm{m}$ width, $4 \mu \mathrm{m}$ height, and $2 \mu \mathrm{m}$ slab thickness, at $\lambda=1.55 \mu \mathrm{m}$. As observed, the mode profile is mainly confined inside the silicon core and the minor component at the corners is quite small. Therefore, both polarization coupling and polarization dependence are weak, as confirmed by the comparison among different BPM results shown in Fig. 2. The normalized propagation constant is defined as:

$$
b=\frac{n_{e f f}^{2}-n_{S_{i O}}^{2}}{n_{S i}^{2}-n_{S i O_{2}}^{2}}
$$

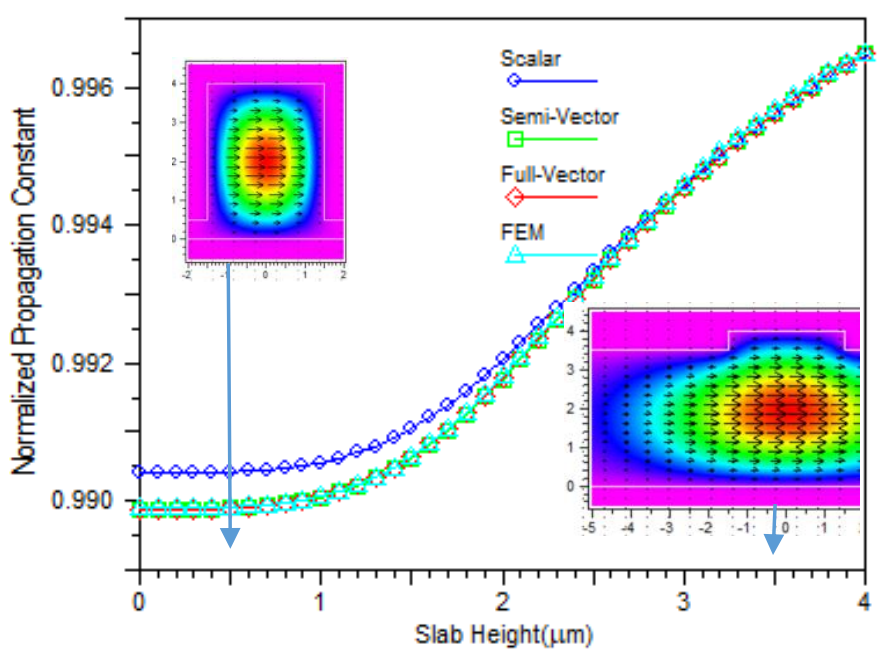

Fig. 2. Normalized propagation constant by different BPMs. Inserted are modal profiles with slab height of $0.5 \mu \mathrm{m}$ and $3.5 \mu \mathrm{m}$.

As an independent check, the rigorous finite-element method (FEM), which is a full-vector solver [11], is also used and the results are shown in the same chart. It is observed:

(1) FV-BPM gives virtually the same results as FEM and its accuracy has been validated against exact analytical solution [9].

(2) SV-BPM yields the similar results as FV-BPM across the whole range, because of the weak minor component of the modal profile.

(3) The discrepancy between scalar and vector results is bigger for the waveguide with smaller slab height because of electric field discontinuities at junctions in the lateral direction. The difference becomes smaller as the slab height increases and eventual vanishes when the rib/ridge becomes a slab.

To maintain single mode conditions, the slab must be $>1.7 \mu \mathrm{m}$ for this example. Therefore, semi-vector or even scalar BPM should be sufficient for large core rib/ridge waveguide.

Instead of absolute phase, the performance of a directional coupler depends on the phase difference between its symmetric and anti-symmetric modes. The coupling length is defined as

$$
L_{c}=\frac{\lambda}{2\left(N_{s}-N_{a}\right)}
$$

where $\mathrm{N}_{\mathrm{s}}$ and $\mathrm{N}_{\mathrm{a}}$ are effective indices of the symmetric and antisymmetric modes of the coupler, respectively.

We calculate the coupling length of a directional coupler consisting of two identical waveguides, which were investigated earlier, and the scan results over edge-to-edge separation are shown in Fig. 3, for different methods.

Similar behaviors are observed, i.e, the semi-vector result is 
very close to the full-vector results of both FV-BPM and FVFEM. Hence, semi-vector simulation is sufficient for directional couplers based on large-core silicon waveguide. At small separations, even scalar BPM is good enough.

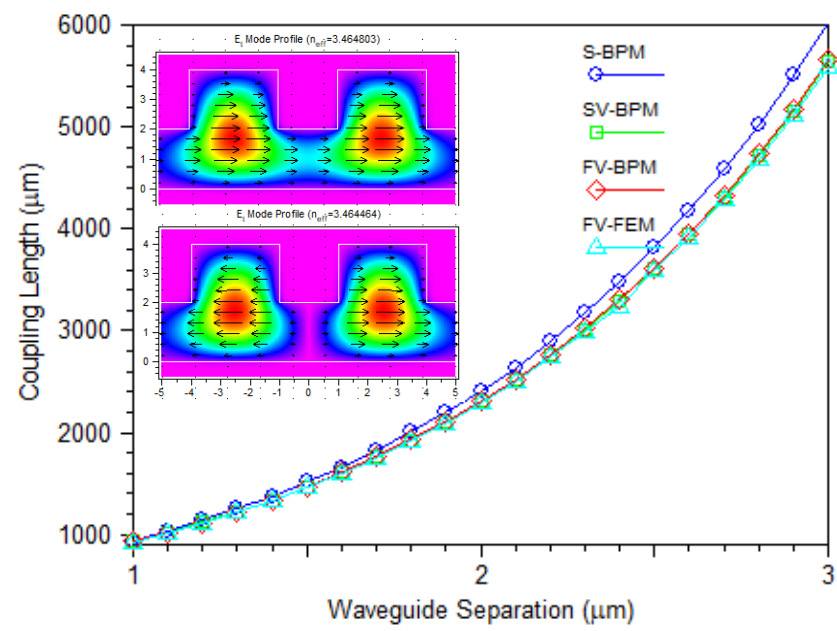

Fig. 3. Coupling length of the directional coupler calculated by different algorithms. Inserted are modal profiles of symmetric and anti-symmetric modes with separation of $2 \mu \mathrm{m}$.

In addition to phase, accurate modeling of the amplitude is equally important for $\mathrm{SiP}$ devices because it directly relates to insertion loss. One of the major contributors to device insertion loss is the waveguide bending loss, which can be calculated from the imaginary part of the effective index $n_{i}$.

$$
I L(d B)=20 \log _{10} e^{-n_{i} k_{0} L}
$$

Since the imaginary-distance method cannot calculate the complex mode, we have to use the correlation method [10] to calculate the loss of a waveguide bend via the conformal mapping technique [12].

To test the accuracy of this approach, we calculate the loss of a $90^{\circ}$ bend using $L=\pi R / 2$ in Eqn. (5). Shown in Fig. 4 are the results at different radii using S-BPM, SV-BPM, and FV-FEM. Instead of FV-BPM, FV-FEM is used because accurate loss calculations require power conservation with scheme parameter $\alpha=0.5$, at which FV-BPM is not stable.

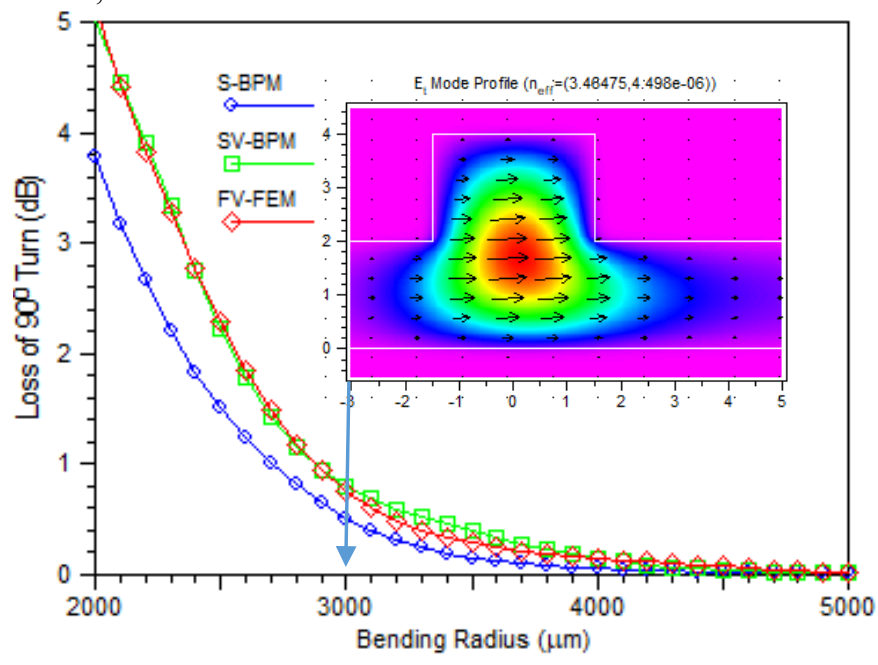

Fig. 4. Bending loss of rib/ridge waveguide calculated by different algorithms. Inserted is the modal profile at bending radius of $3 \mathrm{~mm}$.
Once again, it is observed that the semi-vector result is very close to the full-vector result, hence SV-BPM is sufficient to accurately model the power in rib/ridge waveguide bends. The above loss curves also confirm our earlier claim that large core rib/ridge waveguides are weakly guiding, and the bending radius cannot be too small. As a result, the device size is usually large.

\section{B. Small core silicon wire waveguide}

Although its dimension is small, typically 500nm x 220nm as industry standard, the silicon wire waveguide has strong confinement, as indicated by the calculated bending loss and modal profile shown in Fig. 5. The waveguide can be bent at radii as small as a few micrometers with little bending loss and the field is still strongly confined inside the core even at $\mathrm{R}=1 \mu \mathrm{m}$.

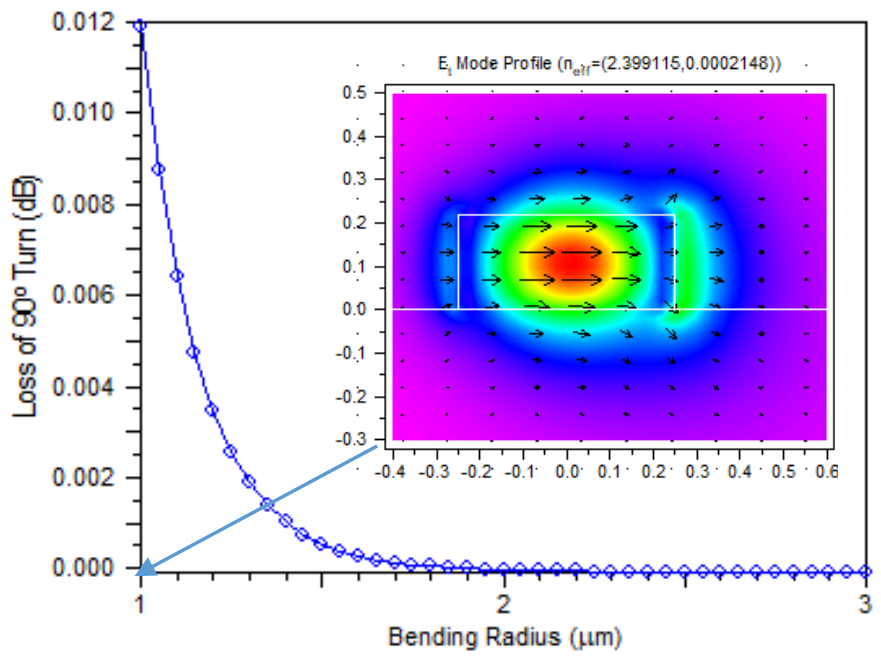

Fig. 5. Bending loss of silicon wire waveguide calculated by FEM. Inserted is the modal profile at bending radius of $1 \mu \mathrm{m}$.

Please note that the above bend mode was calculated by FEM in cylindrical coordinates because BeamPROP BPM only supports conformal mapping, which is valid only when the bend radius is much larger than the structure [12].

Because of its strong confinement, the silicon wire waveguide is strongly polarization dependent and polarization coupled. As shown in Fig. 6, the E-field discontinuity is clearly observed, and the minor component is quite strong.
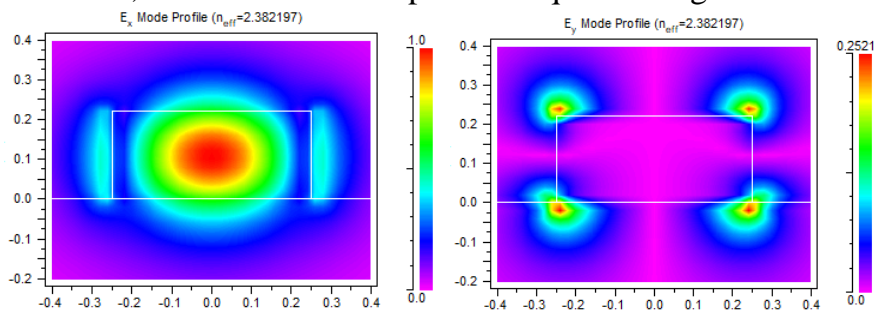

Fig. 6. Major and minor components of silicon wire waveguide mode

However, the polarization dependence and polarization coupling become weaker as the silicon wire waveguide gets wider, as shown in Fig. 7. When the waveguide is wide enough, like a slab waveguide, scalar, semi-vector, and full-vector results become the same. 
The results imply:

- $\mathrm{SV}-\mathrm{BPM}$ is sufficient for phase-insensitive devices, such as adiabatic spot size convertors (SSC).

- For very wide waveguides like star couplers, wellbehaved S-BPM is sufficient.

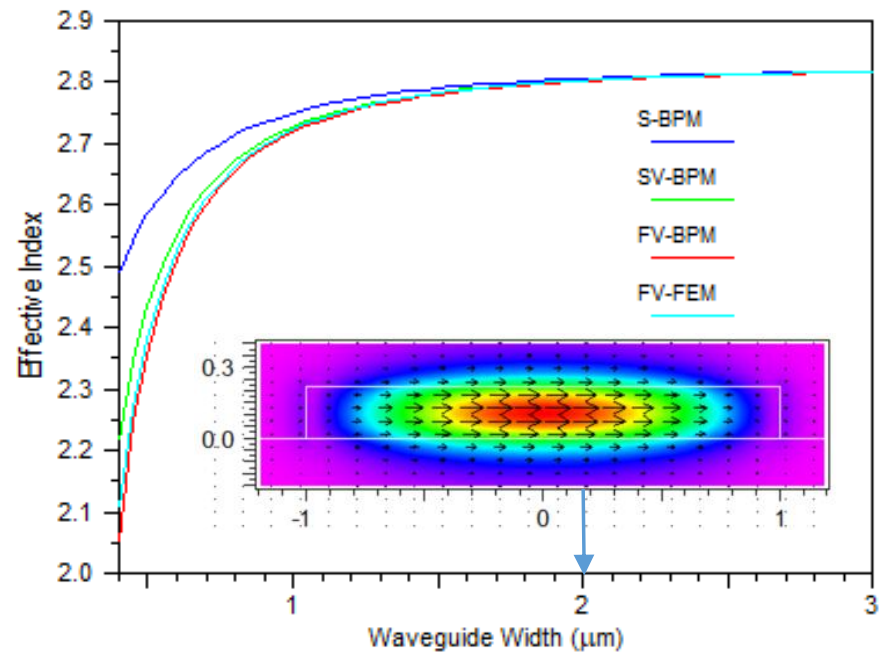

Fig. 7 Effective indices of the fundamental mode of a silicon wire waveguide. Inserted is the modal profile at waveguide width of $2 \mu \mathrm{m}$.

It is true that many $\mathrm{SiP}$ devices are phase sensitive and accurate full-vector simulations may be needed. For some of them, such as directional couplers and MMIs, the performance critically depends on the phase difference between the coupled modes, rather than their absolute phases [13].

To explore the suitability of different BPMs for SiP devices built on silicon wire waveguides, we also calculate the $2^{\text {nd }}$ mode and plot its effective index difference with the fundamental mode in Fig. 8.

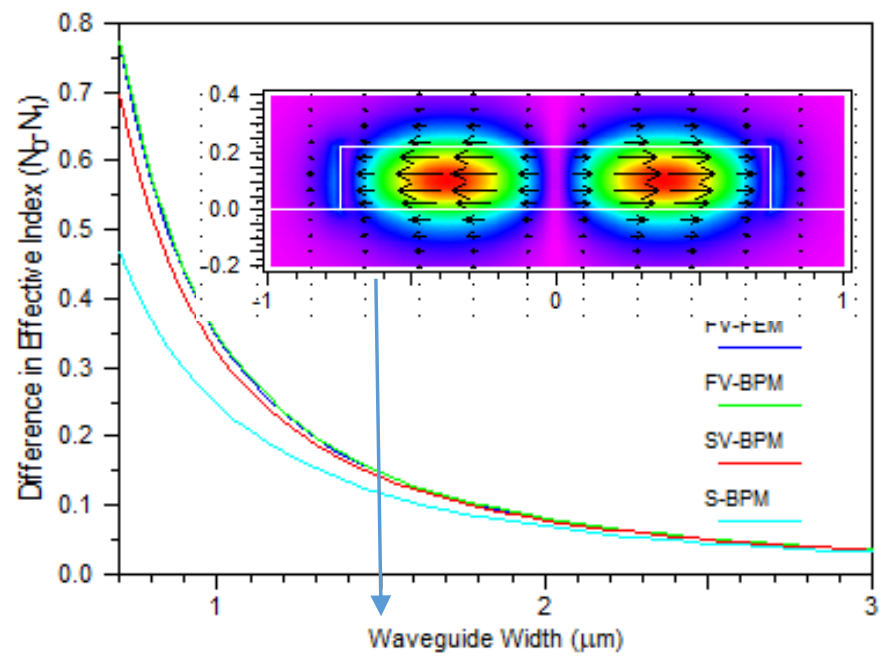

Fig. 8 Difference in effective indices of the $1^{\text {st }}$ and $2^{\text {nd }}$ modes. Inserted is the modal profile of the $2^{\text {nd }}$ mode at waveguide width of $1.5 \mu \mathrm{m}$.

Once again, it is found that (1) the semi-vector result is very close the full-vector one, and (2) the difference gets smaller for wider waveguides. Hence, phase-sensitive devices like directional couplers and MMIs, can be simulated by the more robust semi-vector BPM. For extremely wide devices like AWG star couplers, unconditionally stable scalar BPM can be used.

\section{APPLICATION CASES}

\section{A. Directional Coupler}

The directional coupler is a common building block for photonic integrated circuits (PICs). With input and output Sbends, which also contribute to the coupling, it is hard to determine the required coupling length with the formula shown in equation (7). Therefore, we need to rely on accurate simulation to design the coupling length as well as the bend length. Taking the large-core rib/ridge waveguide studied in previous section as the example, shown in Fig. 9 is the comparison of the power remaining in the input branch, calculated by different algorithms at different coupler lengths for a directional coupler with a $1 \mu \mathrm{m}$ edge-to-edge separation.

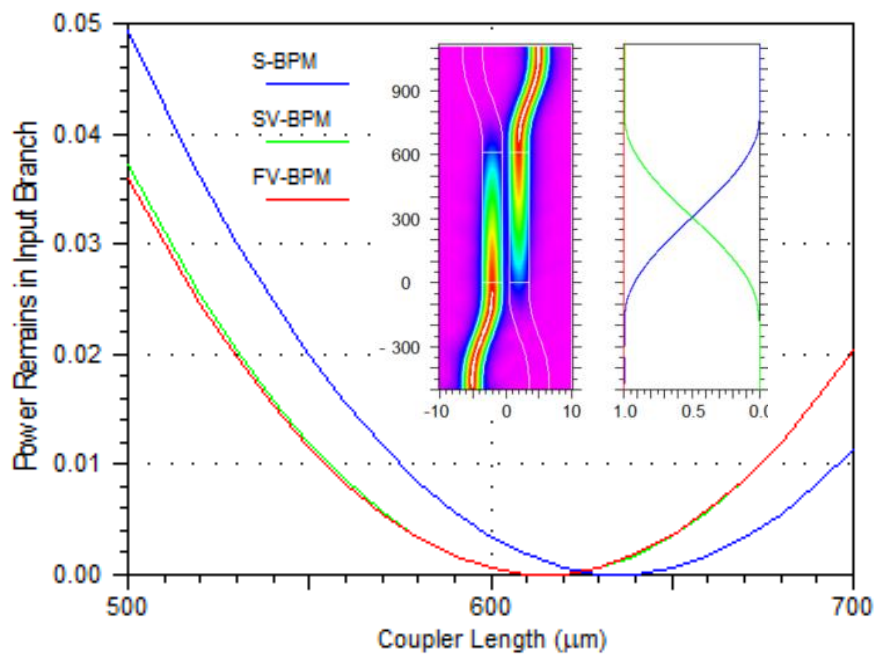

Fig. 9 Power remaining in the input waveguide by different algorithms. Inserted are the simulated field pattern and monitored powers.

Please note that a scheme parameter $\alpha=0.65$ is used for the FV-BPM simulation to keep the algorithm stable. To compensate the artificial loss and calibrate the simulation results, we have done two simulations with exactly the same parameters, such as scheme parameter, domain size, mesh size, device length, etc., except one is with S-bends and the other one with two straight waveguides. In both cases, the artificial loss should be similar, and the extra loss for the coupler with Sbends should be its insertion loss since the straight structure is lossless. We re-normalized the simulation result of the bend structure to the total power of the straight structure. This trick can be applied to other cases for which FV-BPM simulations are needed, such as polarization rotators for which full-vector simulation is a must.

As observed, SV-BPM and FV-BPM give the same coupling length while S-BPM is off by about $20 \mu \mathrm{m}$. This has confirmed our prediction in the previous section, i.e., SV-BPM is sufficient for directional coupler based on large-core rib/ridge waveguide.

We did not compare these results against other algorithms like FDTD or Eigen-Mode Expansion (EME) since the 
structure is fairly large to simulate via those methods in a reasonable time on regular computers.

\section{B. Spot-Size Converter}

Spot-size converters (SSCs) [14] are another key PIC component, used to transform the beam spot from small silicon wires to large core waveguides, which provide better coupling to optical fibers. Because it is adiabatic, an SSC is phase insensitive, hence SV-BPM should be sufficient as mentioned in the previous section. Shown in Fig. 10 is a comparison of the simulated output power by different methods. The SV-BPM and FV-BPM simulations were performed at a large number of different taper lengths; however, the FDTD and EME simulations were performed on fewer taper lengths since they are relatively time consuming.

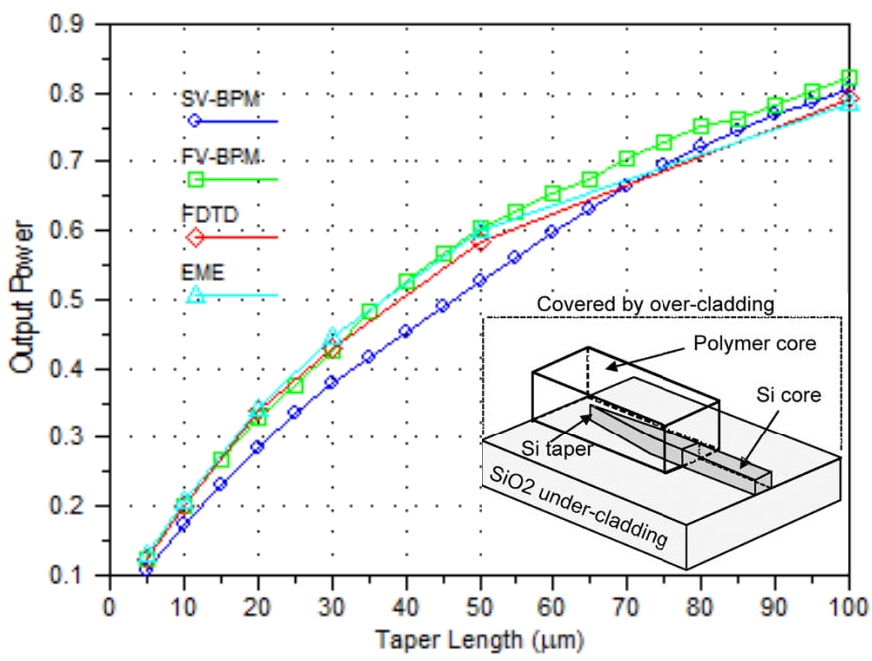

Fig. 10 Simulated output power by different methods. Inserted is the schematic diagram of the SSC in Ref [14] with permission from IEEE.

Please note that the scheme parameter $\alpha=0.55$ is used for FV-BPM simulations to remain stable. The same renormalization trick, used for the directional coupler, is used here.

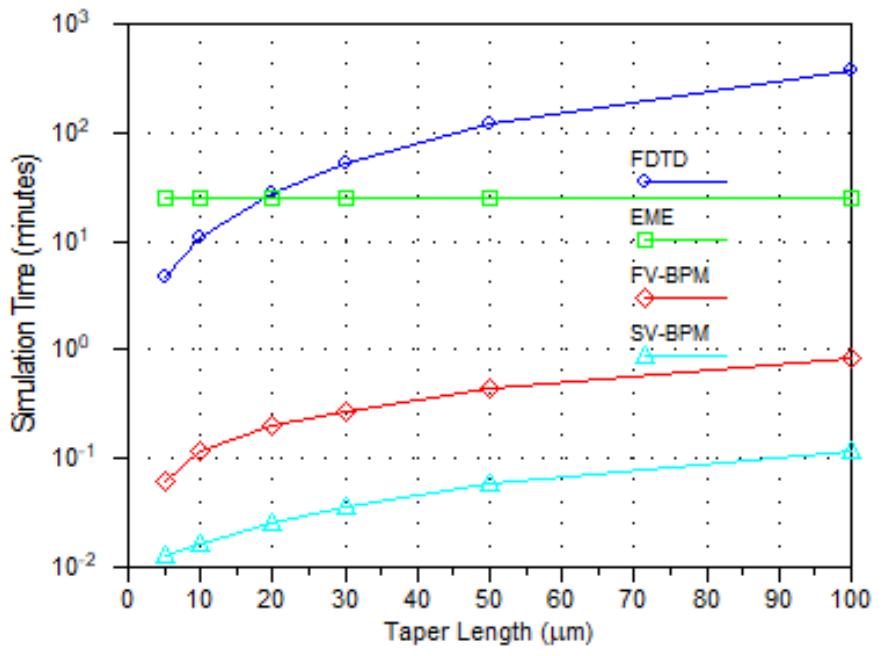

Fig. 11 Computation time by different methods.
As observed, both SV-BPM and FV-BPM give similar results as FDTD and EME, but the simulation time is several orders of magnitude faster than FDTD, esp. for SV-BPM which is 1000+ times faster, as shown in Fig. 11 .

\section{C. $M M I$}

The performance of MMIs is based on mode coupling among different modes and its coupling length is determined by the difference of their effective indices, esp. between the $1^{\text {st }}$ and $2^{\text {nd }}$ modes [13]. Shown in Fig. 12 are the simulation results of both SV-BPM and FDTD for a 2x2 MMI based on silicon wires.

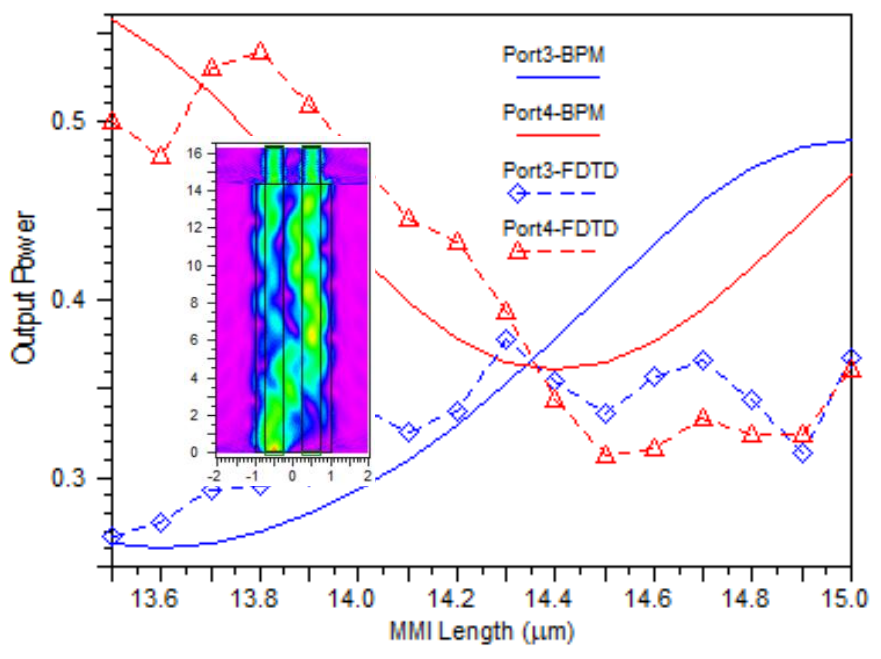

Fig. 12 Simulated output power by different methods. Inserted is the BPM simulated field pattern of the MMI at a length of $14.34 \mu \mathrm{m}$.

As observed, SV-BPM predicts almost the same coupling length as FDTD does, $14.34 \mu \mathrm{m}$ vs $14.37 \mu \mathrm{m}$, but the speed is $1000 \mathrm{x}$ faster for this test case. Please note that wide-angle BPM is used in order to gain high accuracy because there are multiple modes inside the MMI. As predicted in the previous section, FV-BPM also gives the same results (not shown here) as SVBPM because the polarization coupling is very weak for such wide waveguides, as shown in Fig. 7 and Fig. 8.

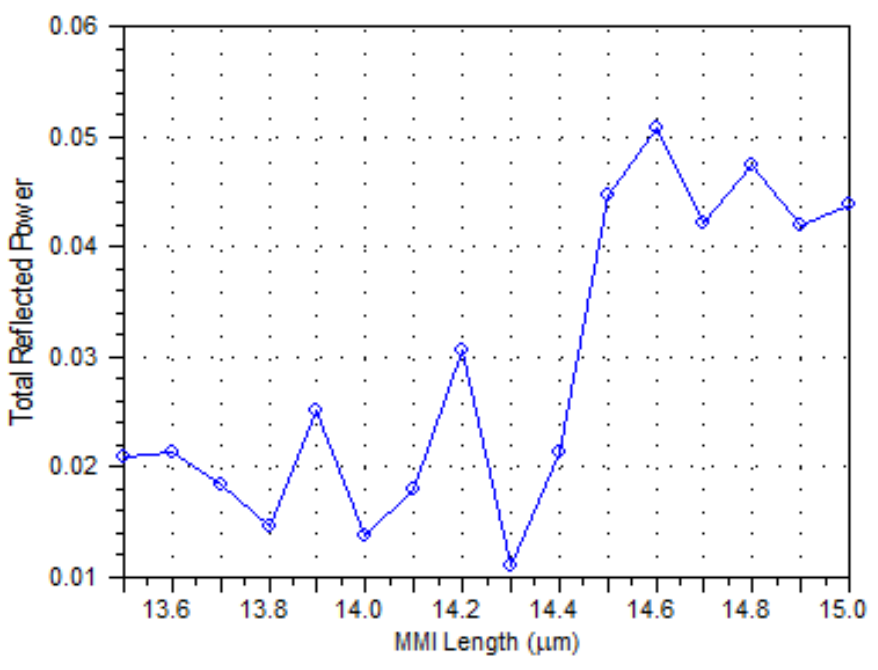

Fig. 13 Total reflected power simulated by FDTD. 
Some discrepancy between BPM and FDTD results is observed when the MMI length is off the optimal coupling length, especially on the longer MMI side. That is because the light focal points are off the port positions and hits silicon-air interface, which generates reflection ignored by BPM. Shown in Fig. 13 is the total reflected power of the MMI device at different MMI length. It shows that the reflection is relatively weak around the optimal length and gets strong off the optimal length, especially on the longer MMI side.

In addition, FDTD results show some oscillation, which is etalon effect because of the Fabry-Perot cavity formed by two MMI junctions.

As an independent check, EME simulation (not shown here) gives the same optimal length $(14.37 \mu \mathrm{m})$ and etalon effect as FDTD.

\section{D. $A W G$}

AWGs are extremely phase-sensitive and have two critical parts, the star coupler and the phase arrays. Because of its large width, the star coupler is virtually a slab waveguide, for which full-vector, semi-vector, or scalar are the same for TE waves. Shown in Fig. 14 are the power outputs from each array waveguide, calculated by BPM and FDTD. As observed, they are very close.

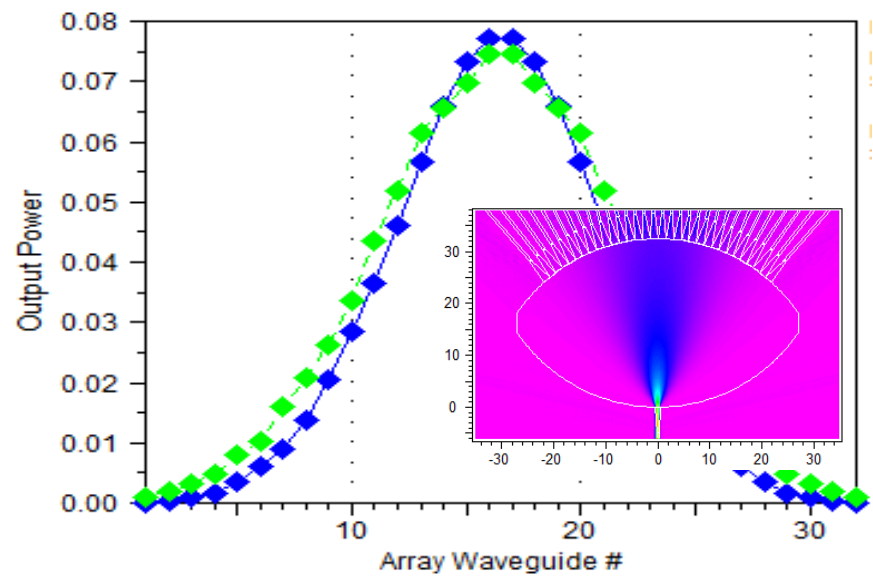

Fig. 14 Simulated output power by BPM (blue) and FDTD (green). Inserted is the BPM simulated field pattern in the input star coupler.

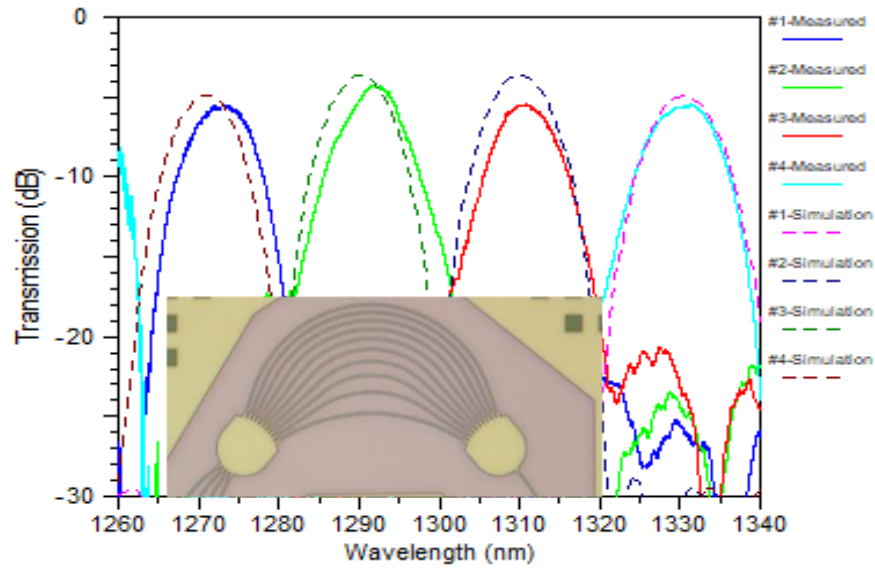

Fig. 15 Comparison between simulation (dashed lines) and measurement (solid lines). Insert is the OM image of the AWG.
Since the effective index of the array waveguides is very critical to determining the FSR and channel position, accurate full-vector modes should be used to simulate the array.

With the above tactics, a 4-channel AWG was designed, simulated, fabricated, and characterized at ITRI. The comparison between simulated and measured results is shown in Fig. 15.

It is observed that the measured spectrum matches the simulation very well, for both FSR and channel positions, as well as for crosstalk level.

\section{CONCLUSION}

In summary, BPM has been proven to be an efficient algorithm for silicon photonics with reasonable accuracy, validated against rigorous FDTD and experimental results. Like any other method, BPM has its own limitations, such as not handling backward reflection from abruptly varying structures. But as long as the assumptions are valid, BPM is suitable for fast prototyping with 1000 times the speed of FDTD. Even in cases where the accuracy may be less than FDTD, optimized designs can be reached quickly, reducing the need for FDTD to only the final few simulations.

\section{REFERENCES}

[1] Huang, Wei-Ping, Chenglin L. Xu, Sai Tak Chu, and Sujeet K. Chaudhuri. "Vector beam propagation method based on finite-difference." In Integrated Optical Circuits, vol. 1583, pp. 268-270. International Society for Optics and Photonics, 1991

[2] Liu, P-L., and B-J. Li. "Study of form birefringence in waveguide devices using the semivectorial beam propagation method." IEEE photonics technology letters 3, no. 10 (1991): 913-915.

[3] Huang, W. P., and C. L. Xu. "Simulation of three-dimensional optical waveguides by a full-vector beam propagation method." IEEE journal of quantum electronics 29, no. 10 (1993): 2639-2649.

[4] Chiou, Yih-Peng, and Hung-chun Chang. "Analysis of optical waveguide discontinuities using the Padé approximants." IEEE Photonics Technology Letters 9, no. 7 (1997): 964-966.

[5] Hadley, G. Ronald. "Wide-angle beam propagation using Padé approximant operators." Optics Letters 17, no. 20 (1992): 1426-1428.

[6] Ma, F., C. L. Xu, and W. P. Huang. "Wide-angle full vectorial beam propagation method." IEE Proceedings-Optoelectronics 143, no. 2 (1996): 139-143.

[7] Xie, Huan, Wangtao Lu, and Ya Yan Lu. "Complex modes and instability of full-vectorial beam propagation methods." Optics letters 36, no. 13 (2011): 2474-2476

[8] BeamPROP BPM, https://www.synopsys.com/photonic-solutions/rsoftphotonic-device-tools/passive-device-beamprop.html.

[9] Xu, C. L., W. P. Huang, and S. K. Chaudhuri. "Efficient and accurate vector mode calculations by beam propagation method." Journal of lightwave technology 11, no. 7 (1993): 1209-1215.

[10] Feit, M. D., and J. A. Fleck. "Computation of mode properties in optical fiber waveguides by a propagating beam method." Applied Optics 19, no. 7 (1980): 1154-1164.

[11] FemSIM FEM, https://www.synopsys.com/photonic-solutions/rsoftphotonic-device-tools/passive-device-femsim.html.

[12] Lui, Wayne W., C-L. Xu, and Wei-Ping Huang. "Full-vectorial wave propagation in semiconductor optical bending waveguides and equivalent straight waveguide approximations." Journal of lightwave technology 16, no. 5 (1998): 910

[13] Smit, Meint K., and Cor Van Dam. "PHASAR-based WDM-devices: Principles, design and applications." IEEE Journal of selected topics in quantum electronics 2, no. 2 (1996): 236-250.

[14] Tsuchizawa, Tai, Koji Yamada, Hiroshi Fukuda, Toshifumi Watanabe, Jun-ichi Takahashi, Mitsutoshi Takahashi, Tetsufumi Shoji, Emi 
Tamechika, Sei-ichi Itabashi, and Hirofumi Morita. "Microphotonics devices based on silicon microfabrication technology." IEEE Journal of selected topics in quantum electronics 11, no. 1 (2005): 232-240.

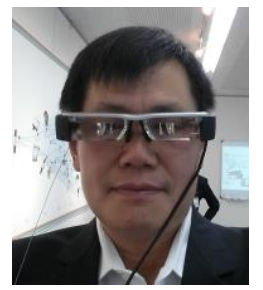

Chenglin Xu (SM'91-M'94) was born in March 10, 1963 in China. He received the BS and MS degrees in optical engineering from Tianjin University, China in 1985 and 1988, and the $\mathrm{PhD}$ degree in electrical engineering from University of Waterloo, Canada in 1994.

Currently, Dr. $\mathrm{Xu}$ is with Synopsys, Inc. where he is the product manager for the RSoft Photonic Device Tools. Before joining RSoft, now a part of Synopsys, in 2007, he was an optical design engineer at JDS Fitel in Ottawa, Canada from 1995 to 1996, the chief scientist and co-founder of Apollo Photonics Inc from 1997 to 2004, and a professor at Shandong University in China from 2005 to 2006. Dr. Xu is the coinventor of vector BPM and has co-authored a book chapter and published more than 60 journal and conference papers. His current research area is application of the photonic device simulation software on various fields, such as photonic integrated circuit, micro-LED, AR/VR, metamaterials, LiDAR, etc.

Dr. $\mathrm{Xu}$ is a member of IEEE and SPIE.

Evan Heller received his Ph.D degree from University of Connecticut in 1998. Currently, he is an R\&D Engineer with Synopsys, Inc. working on the RSoft Photonic Device Tools.

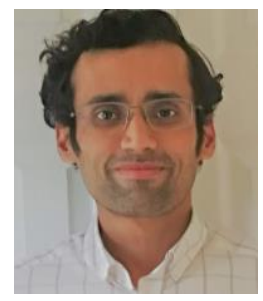

Mayank Bahl received his BTech from IIT Madras and $\mathrm{MS}$ and $\mathrm{PhD}$ degrees in computational photonics from Columbia University. Until 2012, he worked at RSoft, Inc., where he was the primary developer for their commercial photonics design software's, FullWAVE, FDTD and LED utility. Currently, he works at Synopsys, Inc. on various aspects of their commercial Photonic Device Tools including Photonic Device Compiler for silicon photonics based PIC design applications and researching mixed-level simulation methods combining wave optic based techniques like FDTD and RCWA with Monte Carlo ray-based techniques.

Rob Scarmozzino, photograph and biography not available at the time of publication.

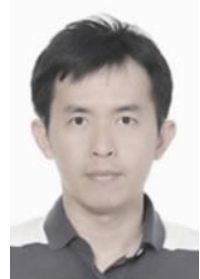

Kai-Ning $\mathrm{Ku}$ received the $\mathrm{Ph} . \mathrm{D}$ degree in institute of photonics technologies from National Tsing-Hua University in 2014. He joined Industrial Technology Research Institute (ITRI) until the present. His interests focus on silicon photonics including device design, process and testing platform development. His work experience includes photonic chip design for high speed optical transceiver and frequency modulation continuous wave (FMCW) LiDAR system.
Ying Zhou, photograph and biography not available at the time of publication.

Tungyu Su, photograph and biography not available at the time of publication.

Po-Chih Chang, photograph and biography not available at the time of publication.

Chen-Yu Lin, photograph and biography not available at the time of publication.

Shang-Chun Chen, photograph and biography not available at the time of publication.

Chih-Lin Wang, photograph and biography not available at the time of publication.

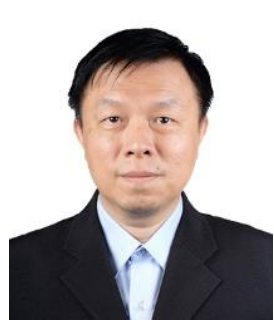

Chien-Chung Lin (S'93-M'02-SM'13) received the B.S. degree in electrical engineering from the National Taiwan University in 1993, and the M.S. and Ph.D. degrees in electrical engineering from Stanford University, Stanford, CA, USA, in 1997 and 2002, respectively. His thesis work focused on design, modeling, and fabrication of micromachined-tunable optoelectronic devices. Starting August in 2021, he joined the Graduate Institute of Photonics and Optoelectronics of National Taiwan University as a full professor. From 2009 to 2021, he was with National Chiao Tung University (NCTU), Tainan, Taiwan, where he held a position as a Professor. The major research efforts in his group are in design and fabrication of novel semiconductor optoelectronic devices, including LEDs, solar cells, and lasers. Since 2016, he has been jointly appointed by the Industrial Technology Research Institute (ITRI), where he participates and leads several key projects in the micro LEDs, 3DIC, and silicon photonics. Before joining NCTU, he worked for different start-ups in the United States. After graduating from Stanford in 2002, he joined E2O Communications Inc., Calabasas, CA, USA, as a Senior Optoelectronic Engineer. His main research interests were in long-wavelength vertical cavity surface emitting lasers. In 2004, he joined Santur Corporation, Fremont, CA, where he had worked on various projects such as monolithic multiwavelength DFB Laser arrays for data and telecommunications applications, yield and reliability analysis of DFB Laser arrays, etc. From 2015 to 2017, he was the recipient of the Young Investigator Research Grant by Ministry of Science and Technology of Taiwan. He has more than 230 journal and conference publications and is a Fellow of the Optical Society (formerly the Optical Society of America) and a senior member of the IEEE Electron Devices Society and Photonics Society. 